Acta Crystallographica Section E

Structure Reports

Online

ISSN 1600-5368

\section{N-(4-Chlorophenyl)morpholine-4- carboxamide}

\section{Yu-Feng Li}

Microscale Science Institute, Department of Chemistry and Chemical Engineering, Weifang University, Weifang 261061, People's Republic of China

Correspondence e-mail: liyufeng8111@163.com

Received 7 August 2011; accepted 23 August 2011

Key indicators: single-crystal X-ray study; $T=293 \mathrm{~K}$; mean $\sigma(\mathrm{C}-\mathrm{C})=0.002 \AA$; $R$ factor $=0.044 ; w R$ factor $=0.124 ;$ data-to-parameter ratio $=17.9$.

In the title molecule, $\mathrm{C}_{11} \mathrm{H}_{13} \mathrm{ClN}_{2} \mathrm{O}_{2}$, the morpholine ring has a chair conformation. In the crystal, molecules are linked into chains along [100] by $\mathrm{N}-\mathrm{H} \cdots \mathrm{O}$ hydrogen bonds.

\section{Related literature}

For the applications of morpholine compounds, see: Arrieta et al. (2007). For related structures, see: Li (2011a,b).

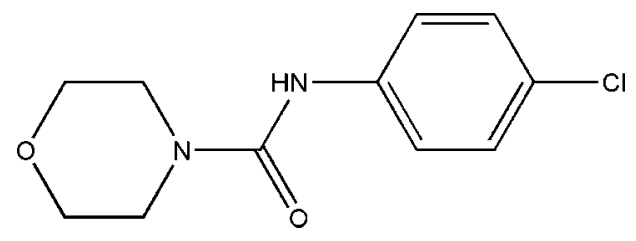

\section{Experimental}

Crystal data

$\mathrm{C}_{11} \mathrm{H}_{13} \mathrm{ClN}_{2} \mathrm{O}_{2}$

$M_{r}=240.68$

Orthorhombic, $\mathrm{Pbca}$

$V=2325.0(8) \AA^{3}$
$Z=8$
Mo $K \alpha$ radiation
Data collection
Bruker SMART CCD
$\quad$ diffractometer
20865 measured reflections
Refinement
$R\left[F^{2}>2 \sigma\left(F^{2}\right)\right]=0.044$
$w R\left(F^{2}\right)=0.124$
$S=1.07$
2660 reflections
149 parameters

$\mu=0.32 \mathrm{~mm}^{-1}$

$T=293 \mathrm{~K}$

$0.26 \times 0.19 \times 0.18 \mathrm{~mm}$

2660 independent reflections 2081 reflections with $I>2 \sigma(I)$

$R_{\text {int }}=0.059$
$\mathrm{H}$ atoms treated by a mixture of independent and constrained refinement
$\Delta \rho_{\max }=0.40$ e $\AA^{-3}$
$\Delta \rho_{\min }=-0.29 \mathrm{e}^{-3}$

Table 1

Hydrogen-bond geometry $\left(\AA,^{\circ}\right)$.

\begin{tabular}{lllll}
\hline$D-\mathrm{H} \cdots A$ & $D-\mathrm{H}$ & $\mathrm{H} \cdots A$ & $D \cdots A$ & $D-\mathrm{H} \cdots A$ \\
\hline $\mathrm{N} 2-\mathrm{H} 1 N \cdots \mathrm{O} 2{ }^{\mathrm{i}}$ & $0.838(19)$ & $2.114(19)$ & $2.9226(19)$ & $162.2(19)$ \\
\hline
\end{tabular}

Symmetry code: (i) $x+\frac{1}{2}, y,-z+\frac{1}{2}$.

Data collection: SMART (Bruker, 1997); cell refinement: SAINT (Bruker, 1997); data reduction: $S A I N T$; $\operatorname{program}(\mathrm{s})$ used to solve structure: SHELXS97 (Sheldrick, 2008); program(s) used to refine structure: SHELXL97 (Sheldrick, 2008); molecular graphics: SHELXTL (Sheldrick, 2008); software used to prepare material for publication: SHELXTL.

Supplementary data and figures for this paper are available from the IUCr electronic archives (Reference: LH5309).

\title{
References
}

Arrieta, A., Otaegui, D., Zubia, A., Cossío, F. P., Díaz-Ortiz, A., Hoz, A., Herrero, A., Prieto, P., Foces-Foces, C., Pizarro, J. L. \& Arriortua, M. I. (2007). J. Org. Chem. 72, 4313-4322.

Bruker (1997). SMART and SAINT. Bruker AXS Inc., Madison, Wisconsin, USA.

Li, Y.-F. (2011a). Acta Cryst. E67, o1796.

Li, Y.-F. (2011b). Acta Cryst. E67, o1792.

Sheldrick, G. M. (2008). Acta Cryst. A64, 112-122. 


\section{supporting information}

Acta Cryst. (2011). E67, o2492 [doi:10.1107/S1600536811034611]

\section{N-(4-Chlorophenyl)morpholine-4-carboxamide}

\section{Yu-Feng Li}

\section{S1. Comment}

Morpholine compounds are an important intermediate reagent in organic synthesis (Arrieta et al., 2007). As part of our search for new carboxamide compounds ( $\mathrm{Li}, 2011 a, b)$ we have determined the crystal structure of the title compound containing a morpholine ring. The molecular structure of the title compound is shown in Fig. 1. The morpholine ring $(\mathrm{N} 1 / \mathrm{C} 1 / \mathrm{C} 2 / \mathrm{C} 3 / \mathrm{C} 4 / \mathrm{O} 1)$ is in a chair conformation. In the crystal, molecules are linked into chains along [100] by $\mathrm{N}-$ $\mathrm{H} \cdots \mathrm{O}$ hydrogen bonds.

\section{S2. Experimental}

A mixture of morpholine ( $0.1 \mathrm{~mol})$, and (4-chlorophenyl)carbamic chloride $(0.1 \mathrm{~mol})$ was stirred in refluxing ethanol (20 $\mathrm{ml}$ ) for $4 \mathrm{~h}$ to afford the title compound $(0.065 \mathrm{~mol}$, yield 65\%). Colourless blocks of the title compound were obtained by recrystallization from ethanol at room temperature.

\section{S3. Refinement}

$\mathrm{H}$ atoms were fixed geometrically and allowed to ride on their attached atoms, with $\mathrm{C}-\mathrm{H}$ distances $=0.93-0.97 \AA$ and with $U_{\text {iso }}(\mathrm{H})=1.2 U_{\mathrm{eq}}(\mathrm{C})$. The $\mathrm{H}$ atom bonded to $\mathrm{N} 2$ was refined independently with an isotropic displacement parameter.

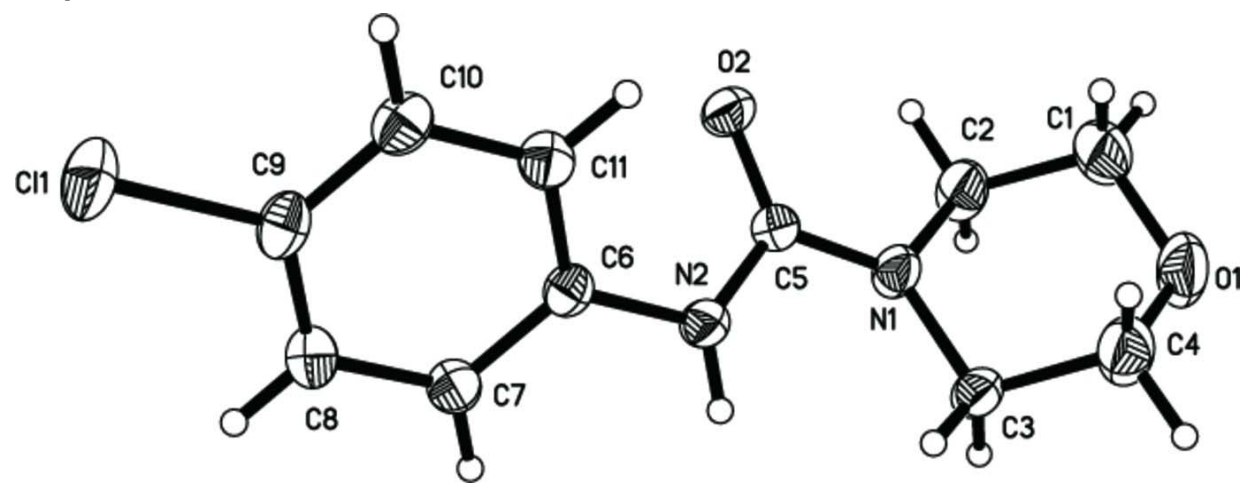

\section{Figure 1}

The molecular structure of the title compound showing $30 \%$ probability displacement ellipsoids.

\section{$N$-(4-Chlorophenyl)morpholine-4-carboxamide}

Crystal data

$\mathrm{C}_{11} \mathrm{H}_{13} \mathrm{ClN}_{2} \mathrm{O}_{2}$

$M_{r}=240.68$

Orthorhombic, $\mathrm{Pbca}$

Hall symbol: -P 2ac 2ab

$a=9.3359$ (19) $\AA$

$$
\begin{aligned}
& b=11.105(2) \AA \\
& c=22.426(5) \AA \\
& V=2325.0(8) \AA^{3} \\
& Z=8 \\
& F(000)=1008
\end{aligned}
$$


$D_{\mathrm{x}}=1.375 \mathrm{Mg} \mathrm{m}^{-3}$

Mo $K \alpha$ radiation, $\lambda=0.71073 \AA$

Cell parameters from 2081 reflections

$\theta=2.6-27.4^{\circ}$

\section{Data collection}

Bruker SMART CCD

diffractometer

Radiation source: fine-focus sealed tube

Graphite monochromator

$\varphi$ and $\omega$ scans

20865 measured reflections

2660 independent reflections

\section{Refinement}

Refinement on $F^{2}$

Least-squares matrix: full

$R\left[F^{2}>2 \sigma\left(F^{2}\right)\right]=0.044$

$w R\left(F^{2}\right)=0.124$

$S=1.07$

2660 reflections

149 parameters

0 restraints

Primary atom site location: structure-invariant direct methods $\mu=0.32 \mathrm{~mm}^{-1}$

$T=293 \mathrm{~K}$

Block, colorless

$0.26 \times 0.19 \times 0.18 \mathrm{~mm}$

2081 reflections with $I>2 \sigma(I)$

$R_{\text {int }}=0.059$

$\theta_{\max }=27.5^{\circ}, \theta_{\min }=3.0^{\circ}$

$h=-11 \rightarrow 12$

$k=-14 \rightarrow 14$

$l=-29 \rightarrow 29$
Secondary atom site location: difference Fourier map

Hydrogen site location: inferred from neighbouring sites

$\mathrm{H}$ atoms treated by a mixture of independent and constrained refinement

$w=1 /\left[\sigma^{2}\left(F_{\mathrm{o}}^{2}\right)+(0.0583 P)^{2}+0.5192 P\right]$ where $P=\left(F_{\mathrm{o}}^{2}+2 F_{\mathrm{c}}{ }^{2}\right) / 3$

$(\Delta / \sigma)_{\max }<0.001$

$\Delta \rho_{\max }=0.40$ e $\AA^{-3}$

$\Delta \rho_{\min }=-0.29$ e $\AA^{-3}$

\section{Special details}

Geometry. All e.s.d.'s (except the e.s.d. in the dihedral angle between two 1.s. planes) are estimated using the full covariance matrix. The cell e.s.d.'s are taken into account individually in the estimation of e.s.d.'s in distances, angles and torsion angles; correlations between e.s.d.'s in cell parameters are only used when they are defined by crystal symmetry. An approximate (isotropic) treatment of cell e.s.d.'s is used for estimating e.s.d.'s involving 1.s. planes.

Refinement. Refinement of $F^{2}$ against ALL reflections. The weighted $R$-factor $w R$ and goodness of fit $S$ are based on $F^{2}$, conventional $R$-factors $R$ are based on $F$, with $F$ set to zero for negative $F^{2}$. The threshold expression of $F^{2}>\sigma\left(F^{2}\right)$ is used only for calculating $R$-factors $(\mathrm{gt})$ etc. and is not relevant to the choice of reflections for refinement. $R$-factors based on $F^{2}$ are statistically about twice as large as those based on $F$, and $R$-factors based on ALL data will be even larger.

Fractional atomic coordinates and isotropic or equivalent isotropic displacement parameters $\left(\AA^{2}\right)$

\begin{tabular}{lllll}
\hline & $x$ & $y$ & $z$ & $U_{\text {iso }} * / U_{\text {eq }}$ \\
\hline C11 & $0.12457(6)$ & $0.60377(5)$ & $0.44436(2)$ & $0.0656(2)$ \\
O1 & $0.41840(19)$ & $0.16232(19)$ & $0.04233(6)$ & $0.0854(6)$ \\
O2 & $0.14298(12)$ & $0.24548(12)$ & $0.21323(5)$ & $0.0505(3)$ \\
N1 & $0.34301(15)$ & $0.19345(14)$ & $0.16222(6)$ & $0.0473(4)$ \\
N2 & $0.35998(15)$ & $0.31812(13)$ & $0.24422(6)$ & $0.0407(3)$ \\
C1 & $0.2740(3)$ & $0.1513(3)$ & $0.06064(9)$ & $0.0782(7)$ \\
H1A & 0.2252 & 0.0940 & 0.0351 & $0.094^{*}$ \\
H1B & 0.2264 & 0.2285 & 0.0567 & $0.094^{*}$ \\
C2 & $0.2651(2)$ & $0.1097(2)$ & $0.12415(8)$ & $0.0598(5)$ \\
H2B & 0.1657 & 0.1058 & 0.1365 & $0.072^{*}$ \\
H2C & 0.3061 & 0.0298 & 0.1277 & $0.072^{*}$ \\
C3 & $0.48960(18)$ & $0.21365(17)$ & $0.14282(7)$ & $0.0477(4)$
\end{tabular}




$\begin{array}{lllll}\text { H3A } & 0.5456 & 0.1411 & 0.1489 & 0.057^{*} \\ \text { H3B } & 0.5322 & 0.2777 & 0.1663 & 0.057^{*} \\ \text { C4 } & 0.4918(2) & 0.2472(2) & 0.07824(9) & 0.0695(6) \\ \text { H4A } & 0.4477 & 0.3257 & 0.0733 & 0.083^{*} \\ \text { H4B } & 0.5903 & 0.2531 & 0.0649 & 0.083^{*} \\ \text { C5 } & 0.27454(16) & 0.25192(14) & 0.20668(7) & 0.0377(3) \\ \text { C6 } & 0.30274(16) & 0.38724(13) & 0.29171(7) & 0.0370(3) \\ \text { C7 } & 0.36139(17) & 0.37550(15) & 0.34817(7) & 0.0416(4) \\ \text { H7A } & 0.4372 & 0.3226 & 0.3545 & 0.050^{*} \\ \text { C8 } & 0.30729(19) & 0.44249(15) & 0.39539(7) & 0.0452(4) \\ \text { H8A } & 0.3465 & 0.4350 & 0.4333 & 0.054^{*} \\ \text { C9 } & 0.19474(19) & 0.52025(14) & 0.38526(7) & 0.0450(4) \\ \text { C10 } & 0.13723(18) & 0.53487(15) & 0.32897(8) & 0.0459(4) \\ \text { H10A } & 0.0626 & 0.5888 & 0.3226 & 0.055^{*} \\ \text { C11 } & 0.19215(18) & 0.46827(14) & 0.28228(7) & 0.0426(4) \\ \text { H11A } & 0.1546 & 0.4779 & 0.2442 & 0.051^{*} \\ \text { H1N } & 0.445(2) & 0.2955(18) & 0.2483(9) & 0.052(5)^{*} \\ \end{array}$

Atomic displacement parameters $\left(\AA^{2}\right)$

\begin{tabular}{lllllll}
\hline & $U^{11}$ & $U^{22}$ & $U^{33}$ & $U^{12}$ & $U^{13}$ & $U^{23}$ \\
\hline C11 & $0.0811(4)$ & $0.0609(3)$ & $0.0549(3)$ & $0.0125(2)$ & $0.0184(2)$ & $-0.0133(2)$ \\
O1 & $0.0807(11)$ & $0.1309(16)$ & $0.0446(7)$ & $-0.0306(11)$ & $0.0110(8)$ & $-0.0221(8)$ \\
O2 & $0.0341(6)$ & $0.0664(8)$ & $0.0511(7)$ & $-0.0016(5)$ & $0.0026(5)$ & $-0.0106(5)$ \\
N1 & $0.0405(7)$ & $0.0582(9)$ & $0.0432(7)$ & $-0.0077(6)$ & $0.0057(6)$ & $-0.0150(6)$ \\
N2 & $0.0347(7)$ & $0.0469(8)$ & $0.0404(7)$ & $0.0043(6)$ & $0.0000(6)$ & $-0.0087(6)$ \\
C1 & $0.0687(14)$ & $0.115(2)$ & $0.0509(12)$ & $-0.0185(14)$ & $-0.0091(11)$ & $-0.0168(12)$ \\
C2 & $0.0559(11)$ & $0.0712(13)$ & $0.0522(10)$ & $-0.0190(9)$ & $0.0065(9)$ & $-0.0229(9)$ \\
C3 & $0.0364(8)$ & $0.0592(10)$ & $0.0475(9)$ & $0.0008(7)$ & $0.0050(7)$ & $-0.0114(8)$ \\
C4 & $0.0668(13)$ & $0.0873(16)$ & $0.0543(11)$ & $-0.0210(12)$ & $0.0089(11)$ & $-0.0037(10)$ \\
C5 & $0.0346(8)$ & $0.0410(8)$ & $0.0375(8)$ & $0.0013(6)$ & $0.0002(6)$ & $0.0008(6)$ \\
C6 & $0.0376(8)$ & $0.0342(8)$ & $0.0391(8)$ & $-0.0014(6)$ & $0.0045(6)$ & $-0.0013(6)$ \\
C7 & $0.0441(9)$ & $0.0378(8)$ & $0.0429(9)$ & $0.0044(6)$ & $-0.0011(7)$ & $-0.0006(6)$ \\
C8 & $0.0537(10)$ & $0.0437(9)$ & $0.0383(8)$ & $-0.0004(7)$ & $0.0031(7)$ & $0.0000(7)$ \\
C9 & $0.0537(9)$ & $0.0356(8)$ & $0.0457(9)$ & $-0.0019(7)$ & $0.0134(8)$ & $-0.0036(6)$ \\
C10 & $0.0488(9)$ & $0.0354(8)$ & $0.0534(10)$ & $0.0062(7)$ & $0.0072(8)$ & $0.0019(7)$ \\
C11 & $0.0475(9)$ & $0.0380(8)$ & $0.0422(8)$ & $0.0033(7)$ & $0.0007(7)$ & $0.0035(6)$ \\
& & & & &
\end{tabular}

Geometric parameters ( $\left.\AA,{ }^{\circ}\right)$

\begin{tabular}{llll}
\hline $\mathrm{C} 11-\mathrm{C} 9$ & $1.7452(16)$ & $\mathrm{C} 3-\mathrm{C} 4$ & $1.496(3)$ \\
$\mathrm{O} 1-\mathrm{C} 1$ & $1.415(3)$ & $\mathrm{C} 3-\mathrm{H} 3 \mathrm{~A}$ & 0.9700 \\
$\mathrm{O} 1-\mathrm{C} 4$ & $1.417(3)$ & $\mathrm{C} 3-\mathrm{H} 3 \mathrm{~B}$ & 0.9700 \\
$\mathrm{O} 2-\mathrm{C} 5$ & $1.2390(19)$ & $\mathrm{C} 4-\mathrm{H} 4 \mathrm{~A}$ & 0.9700 \\
$\mathrm{~N} 1-\mathrm{C} 5$ & $1.351(2)$ & $\mathrm{C} 4-\mathrm{H} 4 \mathrm{~B}$ & 0.9700 \\
$\mathrm{~N} 1-\mathrm{C} 3$ & $1.453(2)$ & $\mathrm{C} 6-\mathrm{C} 7$ & $1.385(2)$ \\
$\mathrm{N} 1-\mathrm{C} 2$ & $1.457(2)$ & $\mathrm{C} 6-\mathrm{C} 11$ & $1.386(2)$ \\
$\mathrm{N} 2-\mathrm{C} 5$ & $1.373(2)$ & $\mathrm{C} 7-\mathrm{C} 8$ & $1.389(2)$
\end{tabular}




\begin{tabular}{|c|c|c|c|}
\hline $\mathrm{N} 2-\mathrm{C} 6$ & $1.4175(19)$ & $\mathrm{C} 7-\mathrm{H} 7 \mathrm{~A}$ & 0.9300 \\
\hline $\mathrm{N} 2-\mathrm{H} 1 \mathrm{~N}$ & $0.84(2)$ & $\mathrm{C} 8-\mathrm{C} 9$ & $1.379(2)$ \\
\hline $\mathrm{C} 1-\mathrm{C} 2$ & $1.499(3)$ & $\mathrm{C} 8-\mathrm{H} 8 \mathrm{~A}$ & 0.9300 \\
\hline $\mathrm{C} 1-\mathrm{H} 1 \mathrm{~A}$ & 0.9700 & $\mathrm{C} 9-\mathrm{C} 10$ & $1.381(3)$ \\
\hline $\mathrm{C} 1-\mathrm{H} 1 \mathrm{~B}$ & 0.9700 & $\mathrm{C} 10-\mathrm{C} 11$ & $1.381(2)$ \\
\hline $\mathrm{C} 2-\mathrm{H} 2 \mathrm{~B}$ & 0.9700 & $\mathrm{C} 10-\mathrm{H} 10 \mathrm{~A}$ & 0.9300 \\
\hline $\mathrm{C} 2-\mathrm{H} 2 \mathrm{C}$ & 0.9700 & $\mathrm{C} 11-\mathrm{H} 11 \mathrm{~A}$ & 0.9300 \\
\hline $\mathrm{C} 1-\mathrm{O} 1-\mathrm{C} 4$ & $110.71(16)$ & $\mathrm{O} 1-\mathrm{C} 4-\mathrm{H} 4 \mathrm{~A}$ & 109.2 \\
\hline $\mathrm{C} 5-\mathrm{N} 1-\mathrm{C} 3$ & $126.33(14)$ & $\mathrm{C} 3-\mathrm{C} 4-\mathrm{H} 4 \mathrm{~A}$ & 109.2 \\
\hline $\mathrm{C} 5-\mathrm{N} 1-\mathrm{C} 2$ & $120.22(14)$ & $\mathrm{O} 1-\mathrm{C} 4-\mathrm{H} 4 \mathrm{~B}$ & 109.2 \\
\hline $\mathrm{C} 3-\mathrm{N} 1-\mathrm{C} 2$ & $113.14(13)$ & $\mathrm{C} 3-\mathrm{C} 4-\mathrm{H} 4 \mathrm{~B}$ & 109.2 \\
\hline $\mathrm{C} 5-\mathrm{N} 2-\mathrm{C} 6$ & $122.11(14)$ & $\mathrm{H} 4 \mathrm{~A}-\mathrm{C} 4-\mathrm{H} 4 \mathrm{~B}$ & 107.9 \\
\hline $\mathrm{C} 5-\mathrm{N} 2-\mathrm{H} 1 \mathrm{~N}$ & $117.2(13)$ & $\mathrm{O} 2-\mathrm{C} 5-\mathrm{N} 1$ & $121.93(14)$ \\
\hline $\mathrm{C} 6-\mathrm{N} 2-\mathrm{H} 1 \mathrm{~N}$ & $116.0(13)$ & $\mathrm{O} 2-\mathrm{C} 5-\mathrm{N} 2$ & $122.28(14)$ \\
\hline $\mathrm{O} 1-\mathrm{C} 1-\mathrm{C} 2$ & $110.80(19)$ & $\mathrm{N} 1-\mathrm{C} 5-\mathrm{N} 2$ & $115.78(13)$ \\
\hline $\mathrm{O} 1-\mathrm{C} 1-\mathrm{H} 1 \mathrm{~A}$ & 109.5 & $\mathrm{C} 7-\mathrm{C} 6-\mathrm{C} 11$ & $119.67(15)$ \\
\hline $\mathrm{C} 2-\mathrm{C} 1-\mathrm{H} 1 \mathrm{~A}$ & 109.5 & $\mathrm{C} 7-\mathrm{C} 6-\mathrm{N} 2$ & $119.12(14)$ \\
\hline $\mathrm{O} 1-\mathrm{C} 1-\mathrm{H} 1 \mathrm{~B}$ & 109.5 & $\mathrm{C} 11-\mathrm{C} 6-\mathrm{N} 2$ & $121.19(14)$ \\
\hline $\mathrm{C} 2-\mathrm{C} 1-\mathrm{H} 1 \mathrm{~B}$ & 109.5 & $\mathrm{C} 6-\mathrm{C} 7-\mathrm{C} 8$ & $120.16(15)$ \\
\hline $\mathrm{H} 1 \mathrm{~A}-\mathrm{C} 1-\mathrm{H} 1 \mathrm{~B}$ & 108.1 & $\mathrm{C} 6-\mathrm{C} 7-\mathrm{H} 7 \mathrm{~A}$ & 119.9 \\
\hline $\mathrm{N} 1-\mathrm{C} 2-\mathrm{C} 1$ & $109.42(17)$ & $\mathrm{C} 8-\mathrm{C} 7-\mathrm{H} 7 \mathrm{~A}$ & 119.9 \\
\hline $\mathrm{N} 1-\mathrm{C} 2-\mathrm{H} 2 \mathrm{~B}$ & 109.8 & $\mathrm{C} 9-\mathrm{C} 8-\mathrm{C} 7$ & $119.14(16)$ \\
\hline $\mathrm{C} 1-\mathrm{C} 2-\mathrm{H} 2 \mathrm{~B}$ & 109.8 & $\mathrm{C} 9-\mathrm{C} 8-\mathrm{H} 8 \mathrm{~A}$ & 120.4 \\
\hline $\mathrm{N} 1-\mathrm{C} 2-\mathrm{H} 2 \mathrm{C}$ & 109.8 & $\mathrm{C} 7-\mathrm{C} 8-\mathrm{H} 8 \mathrm{~A}$ & 120.4 \\
\hline $\mathrm{C} 1-\mathrm{C} 2-\mathrm{H} 2 \mathrm{C}$ & 109.8 & $\mathrm{C} 8-\mathrm{C} 9-\mathrm{C} 10$ & $121.34(15)$ \\
\hline $\mathrm{H} 2 \mathrm{~B}-\mathrm{C} 2-\mathrm{H} 2 \mathrm{C}$ & 108.2 & $\mathrm{C} 8-\mathrm{C} 9-\mathrm{Cl} 1$ & $119.59(14)$ \\
\hline $\mathrm{N} 1-\mathrm{C} 3-\mathrm{C} 4$ & $109.94(15)$ & $\mathrm{C} 10-\mathrm{C} 9-\mathrm{Cl} 1$ & $119.06(14)$ \\
\hline $\mathrm{N} 1-\mathrm{C} 3-\mathrm{H} 3 \mathrm{~A}$ & 109.7 & $\mathrm{C} 11-\mathrm{C} 10-\mathrm{C} 9$ & $119.06(16)$ \\
\hline $\mathrm{C} 4-\mathrm{C} 3-\mathrm{H} 3 \mathrm{~A}$ & 109.7 & $\mathrm{C} 11-\mathrm{C} 10-\mathrm{H} 10 \mathrm{~A}$ & 120.5 \\
\hline $\mathrm{N} 1-\mathrm{C} 3-\mathrm{H} 3 \mathrm{~B}$ & 109.7 & $\mathrm{C} 9-\mathrm{C} 10-\mathrm{H} 10 \mathrm{~A}$ & 120.5 \\
\hline $\mathrm{C} 4-\mathrm{C} 3-\mathrm{H} 3 \mathrm{~B}$ & 109.7 & $\mathrm{C} 10-\mathrm{C} 11-\mathrm{C} 6$ & $120.59(16)$ \\
\hline $\mathrm{H} 3 \mathrm{~A}-\mathrm{C} 3-\mathrm{H} 3 \mathrm{~B}$ & 108.2 & $\mathrm{C} 10-\mathrm{C} 11-\mathrm{H} 11 \mathrm{~A}$ & 119.7 \\
\hline $\mathrm{O} 1-\mathrm{C} 4-\mathrm{C} 3$ & $112.21(18)$ & $\mathrm{C} 6-\mathrm{C} 11-\mathrm{H} 11 \mathrm{~A}$ & 119.7 \\
\hline $\mathrm{C} 4-\mathrm{O} 1-\mathrm{C} 1-\mathrm{C} 2$ & $-60.2(3)$ & $\mathrm{C} 6-\mathrm{N} 2-\mathrm{C} 5-\mathrm{N} 1$ & $177.97(15)$ \\
\hline $\mathrm{C} 5-\mathrm{N} 1-\mathrm{C} 2-\mathrm{C} 1$ & $120.4(2)$ & $\mathrm{C} 5-\mathrm{N} 2-\mathrm{C} 6-\mathrm{C} 7$ & $130.52(17)$ \\
\hline $\mathrm{C} 3-\mathrm{N} 1-\mathrm{C} 2-\mathrm{C} 1$ & $-53.6(2)$ & $\mathrm{C} 5-\mathrm{N} 2-\mathrm{C} 6-\mathrm{C} 11$ & $-51.2(2)$ \\
\hline $\mathrm{O} 1-\mathrm{C} 1-\mathrm{C} 2-\mathrm{N} 1$ & $57.1(3)$ & $\mathrm{C} 11-\mathrm{C} 6-\mathrm{C} 7-\mathrm{C} 8$ & $1.6(2)$ \\
\hline $\mathrm{C} 5-\mathrm{N} 1-\mathrm{C} 3-\mathrm{C} 4$ & $-121.82(19)$ & $\mathrm{N} 2-\mathrm{C} 6-\mathrm{C} 7-\mathrm{C} 8$ & $179.86(15)$ \\
\hline $\mathrm{C} 2-\mathrm{N} 1-\mathrm{C} 3-\mathrm{C} 4$ & $51.7(2)$ & $\mathrm{C} 6-\mathrm{C} 7-\mathrm{C} 8-\mathrm{C} 9$ & $0.1(2)$ \\
\hline $\mathrm{C} 1-\mathrm{O} 1-\mathrm{C} 4-\mathrm{C} 3$ & $58.8(3)$ & $\mathrm{C} 7-\mathrm{C} 8-\mathrm{C} 9-\mathrm{C} 10$ & $-1.6(3)$ \\
\hline $\mathrm{N} 1-\mathrm{C} 3-\mathrm{C} 4-\mathrm{O} 1$ & $-53.6(2)$ & $\mathrm{C} 7-\mathrm{C} 8-\mathrm{C} 9-\mathrm{Cl}$ & $179.34(13)$ \\
\hline $\mathrm{C} 3-\mathrm{N} 1-\mathrm{C} 5-\mathrm{O} 2$ & $166.36(17)$ & $\mathrm{C} 8-\mathrm{C} 9-\mathrm{C} 10-\mathrm{C} 11$ & $1.3(3)$ \\
\hline $\mathrm{C} 2-\mathrm{N} 1-\mathrm{C} 5-\mathrm{O} 2$ & $-6.8(3)$ & $\mathrm{C} 11-\mathrm{C} 9-\mathrm{C} 10-\mathrm{C} 11$ & $-179.61(13)$ \\
\hline $\mathrm{C} 3-\mathrm{N} 1-\mathrm{C} 5-\mathrm{N} 2$ & $-14.0(2)$ & $\mathrm{C} 9-\mathrm{C} 10-\mathrm{C} 11-\mathrm{C} 6$ & $0.4(3)$ \\
\hline $\mathrm{C} 2-\mathrm{N} 1-\mathrm{C} 5-\mathrm{N} 2$ & $172.86(16)$ & $\mathrm{C} 7-\mathrm{C} 6-\mathrm{C} 11-\mathrm{C} 10$ & $-1.8(2)$ \\
\hline $\mathrm{C} 6-\mathrm{N} 2-\mathrm{C} 5-\mathrm{O} 2$ & $-2.4(2)$ & $\mathrm{N} 2-\mathrm{C} 6-\mathrm{C} 11-\mathrm{C} 10$ & $179.88(15)$ \\
\hline
\end{tabular}


supporting information

Hydrogen-bond geometry $\left(\AA,{ }^{\circ}\right)$

\begin{tabular}{lllll}
\hline$D-\mathrm{H} \cdots A$ & $D-\mathrm{H}$ & $\mathrm{H} \cdots A$ & $D \cdots A$ & $D-\mathrm{H}^{\cdots} \cdot A$ \\
\hline $\mathrm{N} 2-\mathrm{H} 1 N \cdots \mathrm{O} 2^{\mathrm{i}}$ & $0.838(19)$ & $2.114(19)$ & $2.9226(19)$ & $162.2(19)$ \\
\hline
\end{tabular}

Symmetry code: (i) $x+1 / 2, y,-z+1 / 2$. 